\title{
The tolerability of single low dose primaquine in glucose-6-phosphate deficient and normal falciparum-infected Cambodians
}

Lek Dysoley ${ }^{1,2}$, Saorin Kim³ ${ }^{3}$, Sergio Lopes ${ }^{4}$, Nimol Khim ${ }^{3}$, Steven Bjorges ${ }^{5}$, Samphornarann Top ${ }^{5}$, Chea Huch', Huy Rekol ${ }^{1}$, Nelli Westercamp ${ }^{6}$, Mark M. Fukuda ${ }^{7}$, Jimee Hwang ${ }^{8}$, Arantxa Roca-Feltrer ${ }^{4}$, Mavuto Mukaka ${ }^{9,10}$, Didier Menard ${ }^{3,11+}$ and Walter R. Taylor ${ }^{9,10^{*}+}$

\begin{abstract}
Background: The WHO recommends single low-dose primaquine (SLDPQ, $0.25 \mathrm{mg} / \mathrm{kg}$ body weight) in falciparuminfected patients to block malaria transmission and contribute to eliminating multidrug resistant Plasmodium falciparum from the Greater Mekong Sub region (GMS). However, the anxiety regarding PQ-induced acute haemolytic anaemia in glucose-6-phosphate dehydrogenase deficiency (G6PDd) has hindered its use. Therefore, we assessed the tolerability of SLDPQ in Cambodia to inform national policy.

Methods: This open randomised trial of dihydroartemisinin-piperaquine (DHAPP) + SLDPQ vs. DHAPP alone recruited Cambodians aged $\geq 1$ year with acute uncomplicated $P$. falciparum. Randomisation was 4:1 DHAPP +SLDPQ: DHAPP for G6PDd patients and 1:1 for G6PDn patients, according to the results of the qualitative fluorescent spot test. Definitive G6PD status was determined by genotyping. Day (D) 7 haemoglobin $(\mathrm{Hb})$ concentration was the primary outcome measure.
\end{abstract}

Results: One hundred nine patients (88 males, 21 females), aged 4-76years (median 23) were enrolled; 12 were G6PDd Viangchan (9 hemizygous males, 3 heterozygous females). Mean nadir Hb occurred on D7 [11.6 (range 6.4 - 15.6) $\mathrm{g} / \mathrm{dL}$ ] and was significantly lower $(p=0.040)$ in G6PDd $(n=9)$ vs. G6PDn $(n=46)$ DHAPP+SLDPQ recipients: 10.9 vs. $12.05 \mathrm{~g} / \mathrm{dL}, \Delta=-1.15$ (95\% Cl: $-2.24-0.05) \mathrm{g} / \mathrm{dL}$. Three G6PDn patients had D7 Hb concentrations < $8 \mathrm{~g} /$ $\mathrm{dL}$; D7-D0 Hbs were $6.4-6.9,7.4-7.4$, and $7.5-8.2 \mathrm{~g} / \mathrm{dL}$.

For all patients, mean (range) D7-D0 Hb decline was $-1.45(-4.8-2.4) \mathrm{g} / \mathrm{dL}$, associated significantly with higher D0 Hb, higher D0 parasitaemia, and receiving DHAPP; G6PDd was not a factor. No patient required a blood transfusion.

Conclusions: DHAPP+SLDPQ was associated with modest Hb declines in G6PD Viangchan, a moderately severe variant. Our data augment growing evidence that SLDPQ in SE Asia is well tolerated and appears safe in G6PDd patients. Cambodia is now deploying SLDPQ and this should encourage other GMS countries to follow suit.

Trial registration: The clinicaltrials.gov reference number is NCT02434952.

Keywords: Malaria, Transmission blocking, Primaquine, G6PD deficiency, Cambodia

\footnotetext{
* Correspondence: bob@tropmedres.ac

${ }^{\dagger}$ Didier Menard and Walter R. Taylor contributed equally to this work.

${ }^{9}$ Mahidol Oxford Tropical Medicine Research unit (MORU), 420/60 Rajvithi

Road, Bangkok 10400, Thailand

${ }^{10}$ Centre for Tropical Medicine, Nuffield Department of Medicine, University

of Oxford, Oxford, UK

Full list of author information is available at the end of the article
}

(c) The Author(s). 2019 Open Access This article is distributed under the terms of the Creative Commons Attribution 4.0 International License (http://creativecommons.org/licenses/by/4.0/), which permits unrestricted use, distribution, and reproduction in any medium, provided you give appropriate credit to the original author(s) and the source, provide a link to the Creative Commons license, and indicate if changes were made. The Creative Commons Public Domain Dedication waiver (http://creativecommons.org/publicdomain/zero/1.0/) applies to the data made available in this article, unless otherwise stated. 


\section{Background}

Despite the 2012 WHO recommendation to add single low dose primaquine (SLDPQ, $0.25 \mathrm{mg} / \mathrm{kg}$ body weight) to artemisinin-based combination therapies (ACTs) for blocking the transmission of Plasmodium falciparum in sick patients, few countries have introduced SDLPQ in their treatment guidelines. Reluctance has centred on the risk of precipitating dose-dependent, acute haemolytic anaemia (AHA) in patients with glucose-6-phosphate dehydrogenase deficiency (G6PDd), the logistical difficulty in identifying G6PDd patients at the point of care, and the lack of suitable, paediatric PQ tablet strengths and formulations [1].

PQ's oxidative metabolites induce oxidant stress leading to damage of red blood cell membranes and acute extra- and intravascular haemolysis [2-4]. AHA is more severe in the more severe G6PDd variants like Viangchan, Mahidol, Coimbra, Canton, Kaiping that are found in SE Asia [5-7], and the Mediterranean variant, found in the Middle East [8]. Moreover, because G6PD hemizygous males and homozygous females have the lowest G6PD enzyme activities, they are most prone to AHA [9]. The risk of clinically significant AHA, which may require blood transfusion, is in the first week when most malaria patients and healthy subjects given $P Q$ reach their nadir haemoglobin $(\mathrm{Hb})$ concentration [10-14].

The safety evidence base of SLDPQ in G6PDd individuals is growing, including an ongoing trial in falciparuminfected African children (ISRCTN11594437). The mild A- $(202,376)$ G6PD variant predominates in Africa [8, 15] and this limits the generalisability of $\mathrm{Hb}$ dynamic data to SE Asia. Nevertheless, several studies demonstrate a common theme. When different PQ doses $(0.25,0.4$ or $0.75 \mathrm{mg} / \mathrm{kg}$ ) are given with ACTs, there is a greater initial fall in mean $\mathrm{Hb}$ concentrations in both symptomatic and asymptomatic, G6PDd malaria infected patients and G6PDd healthy individuals compared to G6PD normal (n) patients and individuals [16-22].

SLDPQ data from SE Asia are limited. One study demonstrated reassuringly modest $\mathrm{Hb}$ declines in healthy Burmese individuals of all ages with 'good' mean baseline $\mathrm{Hb}$ concentrations [10.8 (< 5y), $11.6(6-15 \mathrm{y})$, 13.4 (adult males) and 11.7 (adult females) g/dL] who underwent mass drug administration with SLDPQ. Mean (upper $95 \%$ confidence interval) fractional falls of 5 (9) \% and 1.7 (4) \% were seen in the G6PDd and G6PDn individuals, respectively [23].

In falciparum-infected, Cambodian adults, $45 \mathrm{mg}$ of PQ $(0.75 \mathrm{mg} / \mathrm{kg})$ was given after dihydroartemisininpiperaquine (DHAPP) at $72 \mathrm{~h}$ [Day (D) 3] and showed a possible trend $(p=0.14)$ of a greater fractional D7-D0 $\mathrm{Hb}$ decline in six G6PDd DHAPP+PQ vs. two G6PDd DHAPP recipients; maximal declines were 29.8 and $4.4 \%$, respectively [24]. Weekly PQ $(0.75 \mathrm{mg} / \mathrm{kg} / \mathrm{w} \mathrm{x} 8 \mathrm{w})$ was well tolerated in 18 G6PDd and 57 G6PDn Cambodians with vivax malaria [12]. Median nadir Hb concentrations occurred on D2 in both groups, rising on D3 (G6PDn) and D7 (G6PDd); median (range) G6PDd vs. G6PDn D7-D0 Hb declines were - $2.2(-4.9-0.8)$ vs. $-0.5(-2.2-2.8) \mathrm{g} / \mathrm{dL}(p=0.0002)$. One G6PDd male was transfused for symptomatic anaemia of $7.2 \mathrm{~g} / \mathrm{dL}$ (D0 $\mathrm{Hb} \quad 10 \mathrm{~g} / \mathrm{dL}$ ). Methaemoglobin (metHb) increases, another dose- and oxidant-related toxicity of $\mathrm{PQ}$, were modest, reaching a maximum of $4.9 \%$. Smithuis et al gave single high dose $P Q, 0.75 \mathrm{mg} / \mathrm{kg}$, to 397 malaria infected patients of all ages who were treated with four ACTs in Myanmar where the moderately severe Mahidol variant has a prevalence of $\sim 10 \%$ [6]; PQ was well tolerated but G6PD status not determined and $\mathrm{Hb}$ measured only on D0 and D63 [25].

The study by Kheng et al [12] encouraged the Cambodian National Malaria Control program (CNM) to conduct a trial of SLDPQ tolerability in uncomplicated falciparum patients to inform drug policy.

\section{Methods \\ Study site}

The study took place from February 2015 to August 2016 in Banlung, Ratanakiri province, NE Cambodia, an area of mixed falciparum vivax transmission. From 2010 to 14, $P$. falciparum $(P f)$ cases fell from $\sim 69-\sim 16 /$ 1000 population and $P$. vivax $(P v)$ from $\sim 16-\sim 11 /$ 1000 [26].

G6PD Viangchan is the predominant variant in Cambodia, occurring at rates of $\sim 15$ and $9 \%$ in western and eastern Cambodia, respectively. Measured enzyme activities in hemizygous males and homozygous females are $\sim 0.5-<3.6 \mathrm{U} / \mathrm{g} \mathrm{Hb}$, equivalent to $\sim 4.1-<30 \%$ of $12 \mathrm{U} / \mathrm{g} \mathrm{Hb}$, the G6PDn population median [7, 27]. Haemoglobin $\mathrm{E}$ is common, $\sim 40 \%$ prevalence, and occurs equally in $P f$ - and $P v$-infected patients [27].

\section{Study design \& participants}

This was a randomised open, parallel safety trial. The inclusion criteria were patients aged $\geq 1$ year $(\mathrm{y})$ and weighing $\geq 7 \mathrm{~kg}$ with acute $(\leq 48 \mathrm{~h})$, symptomatic $\left(\geq 38^{\circ}\right.$ $\mathrm{C}$ axilla $/ \geq 37.5^{\circ} \mathrm{C}$ aural $/$ history of fever), uncomplicated falciparum malaria ( $\geq 1$ asexual form/500 white blood cells) who or whose legal guardian gave signed informed consent and children aged age 12 to $<18$ y signed an assent form.

Excluding criteria were: (i) clinical signs of severe malaria/danger signs, (ii) $\mathrm{Hb}<5 \mathrm{~g} / \mathrm{dL}$ or $<6 \mathrm{~g} / \mathrm{dL}+$ significant anaemia-related symptoms, (iii) pregnant/ breast feeding, (iv) unable/unwilling to take a pregnancy test (women of child-bearing age), (v) intentional pregnancy in coming 3 months, (vi) allergic to PQ/DHAPP, (vii) on known haemolysing drugs in G6PDd, (viii) a 
significant concurrent illness/infection $+/-$ treatment e.g. HIV, TB treatment, steroids, and (ix) on drugs influencing $\mathrm{PQ} / \mathrm{DHAPP}$ pharmacokinetics e.g., antiretrovirals, cimetidine, antiepileptics.

The Cambodian National Ethics Committee approved the study, which also underwent human subjects review at the U.S. Centers for Disease Control and Prevention and was approved as non-engagement in human subjects research. The trial registration reference is NCT02434952.

\section{Conduct of clinical trial Interventions}

All enrolled patients were randomised to receive DHAPP (Eurartesim ${ }^{\circ}$, Sigma Tau, Pomezia, Italy), dosed by weight following the manufacturer's instructions; in July, the dose was changed for children $<25 \mathrm{~kg}$, following new WHO guidelines, so that they received a DHA target dose of $2.5 \mathrm{mg} / \mathrm{kg}$. This dosing change affected 10 children: 5 received the Sigma Tau dose and 5 the WHO dose. Only the largest DHAPP tablet strength (DHA 40 mg, PP $320 \mathrm{mg}$ ) was available in Cambodia, necessitating the use of tablet fractions.

Primaquine tablets, containing $15 \mathrm{mg}$ of PQ base (Government Pharmaceutical Organisation, Bangkok, Thailand), were dosed by weight. The target dose was $0.25 \mathrm{mg}$ base $/ \mathrm{kg}$ and tablet fractions were used as needed. For small children, PQ tablets were dissolved in $5 \mathrm{~mL}$ of water $(3 \mathrm{mg} / \mathrm{mL})$ and dosed accordingly. Patients were observed for early $(\leq 1 \mathrm{~h})$ vomiting and a full or half dose of both drugs were readministered within 30 and $60 \mathrm{~min}$, respectively.

\section{Evaluations}

Patients were admitted to the research clinic for the first 3 days for supervised treatment and investigations. Baseline procedures included a history of illness, recent drug intake, family history, symptom questionnaire, physical examination, and laboratory evaluations. Follow up was limited to 28 days because the focus of this safety study was the initial fall in and early recovery of $\mathrm{Hb}$.

On study Days (D) 0, 1, 2, 3, 7, 14, and 28, the following were done: (i) symptom checklist, (ii) vital signs, metHb [transcutaneous Masimo RAD 57 oximeter (Masimo, Irvine, CA)], (iii) $\mathrm{Hb}$ (HemoCue ${ }^{\ominus}$ Angelholm, Sweden), (iv) Giemsa-stained malaria blood films, read under $\times 1000$ magnification; a negative thick film was declared after reading 8000 white blood cells, (v) thin blood films for red cell morphology and reticulocyte count, and (vi) dried blood spots for: (a) malaria genotype (msp1, msp2, glurp genes) to differentiate recurrent parasitaemia, (b) PQ concentrations (D0, + $1 \mathrm{~h},+2 \mathrm{~h},+$ $8 \mathrm{~h},+12 \mathrm{~h} \&+24 \mathrm{~h}$ ), and (c) piperaquine concentrations (D0, D7, D of recurrent parasitaemia).
Other samples taken were for: (i) in vitro parasite drug sensitivity (D0), (ii) full blood count: D0, 7, 14, 28 [ABX Pentra XL 80 (Horiba Medical, Irvine, CA], (iii) $\mathrm{Hb}$ (WHO Hb colour chart: D0, 7, 14, 28), and (iv) biochemistry (D0 \& 28), using a Pentra C400 (Horiba Medical, Irvine, CA).

G6PD status in the field was diagnosed initially using the fluorescent spot test (FST) to allow for the allocation of SLDPQ (see Randomisation below). The qualitative, Carestart $^{\bullet}$ (AccessBio, Somerset, NJ), rapid diagnostic test (RDT) was assessed in parallel. Both tests detect reliably enzyme activities $<30 \%$ of a normal male population and have a high degree of concordance [7, 28].

G6PD enzyme activity was measured by the Trinity Biotech quantitative G6PD assay ${ }^{\mathrm{Tm}}$ (Trinity Biotech, Jamestown, NY), adapted on an Integra 400 analyser $^{\text {тм }}$ (Roche Diagnostic, Basel, Switzerland)]. Fresh samples were sent on ice from the site to Phnom Penh where the $\mathrm{Hb}$ type was also determined by $\mathrm{Hb}$ electrophoresis (MINICAP system $^{\mathrm{Tm}}$, Sebia, Evry, France).

\section{G6PD genotyping}

Common SE Asian G6PD gene mutations were sought in selected patients with G6PD enzyme activities $\leq 12.3$ $\mathrm{U} / \mathrm{g} \mathrm{Hb}$ (i.e. just above the normal population median of $12 \mathrm{U} / \mathrm{g} \mathrm{Hb}$ [7]): (i) in exon 6 for the Mahidol (487G > A), Mediterranean $(563 \mathrm{C}>\mathrm{T})$ and Coimbra $(592 \mathrm{C}>\mathrm{T})$ variants, (ii) in exon 9 for the Viangchan (871G $>$ A) and Chinese-5 $(1024 \mathrm{C}>\mathrm{T})$ variants, (iii) in exon 11 for the Union $(1360 \mathrm{C}>\mathrm{T})$ variant, and (iv) in exon 12 for the Canton (1376G $>\mathrm{T})$ variant. All other patients with G6PD activity $>12.3 \mathrm{U} / \mathrm{g} \mathrm{Hb}$ were classed as G6PD wild type (normal).

\section{Outcomes}

The primary outcome was the D7 $\mathrm{Hb}$ concentration. The main secondary outcomes included: (i) D7-D0 absolute and fractional falls, (ii) modelled $\mathrm{Hb}$ changes over time, (iii) total malaria attributable fall (MAFt): $\mathrm{D} 28 \mathrm{Hb}$-nadirHb, (iv) $\mathrm{Hb}$ recovery (D28 $\mathrm{Hb}>\mathrm{D} 0 \mathrm{Hb}$ concentration), (v) G6PD geno- and phenotype, thalassemia type, [29]), (vi) D28 cure rate, (vii) gametocyte carriage, and (viii) PQ, carboxyPQ, and PP concentrations.

\section{Tolerability, adverse events (AEs) and SAEs}

These were assessed by an open question, symptom checklist, and a visual analogue scale graded 1 ("I/my child feel/s very bad") to 5 ("I/my child feel/s very well"). We recorded the following AEs and SAEs: (i) early $(\leq 1$ h) or late $(>1 \mathrm{~h}$ ) abdominal pain, nausea or vomiting, (ii) $\mathrm{Hb}$ fractional fall $\geq 25 \%$ vs. D0, (iii) passing dark urine graded $\geq 5$ (Hillmen colour chart [29]), (iv) itching, (v) rash, (vi) AEs of concern (physician judgement), and (vii) any drug-related SAEs. AEs were graded using the 
2004 Division of AIDS toxicity table for adults and children (https://rsc.tech-res.com/docs/default-source/safety /table_for_grading_severity_of_adult_pediatric_adverse_ events.pdf).

\section{Randomisation \& blinding}

Separate computer-generated randomisation lists were used for the G6PDd and G6PDn groups. G6PDd patients were randomised 4:1 to receive DHAPP+SLDPQ and DHAPP (i.e. 4 DHAPP+SLDPQ for every one DHAPP); the G6PDn group was randomised on a 1:1 basis. The randomisation lists showed the treatment allocation (i.e. DHAPP+SLDPQ or DHAPP) and were openly accessible to the senior research team member responsible for drug allocation.

\section{Sample size calculation}

The mean [standard deviation (SD)] D7 $\mathrm{Hb}$ in $\sim 6800$ ACT-treated, falciparum-infected patients of all ages from SE Asia, was 11.27 (1.74) g/dL (WRJT, unpublished). For a two-sided alpha of 0.05 , power of $80 \%$, SD of $1.74 \mathrm{~g} / \mathrm{dL}$, and assuming mean $\mathrm{D} 7 \mathrm{Hb} \Delta$ of $-1 \mathrm{~g} / \mathrm{dL}$ in the G6PDd DHAPP+SLDPQ arm vs. G6PDn DHAPP + SLDPQ arm, the sample size was 48/arm, rounded up to 50. We also decided to recruit 50 G6PDd and 50 G6PDn patients to receive DHAPP ('control' group).

\section{Data management and statistical methods}

Data collected on paper case record forms were double entered into Epi Data v3.1, cleaned, then analysed in Stata v14 (Stata Corporation, College Station, TX). All patients who received $\geq 1$ dose of DHAPP +/- SLDPQ were analysed.

ABX Pentra XL 80 measured Hb concentrations were considered the 'gold' standard and used for analyses involving D0, D7 and D7-D0 declines. Because HemoCue $\mathrm{Hb}$ measurements were more frequent, we used these results for: (i) defining the $\mathrm{Hb}$ nadir day, (ii) modelling $\mathrm{Hb}$ dynamics, (iii) MAFt, and (iv) $\mathrm{Hb}$ recovery, cognizant that HemoCue $\mathrm{Hb}$ concentrations were generally higher than those of the ABX Pentra XL 80 .

Proportional data were compared using chi-squared or Fisher's exact test, as appropriate. Student's ' $t$ ' and non-parametric tests were used to assess mean and distribution differences in normally or skewed distributed continuous data, respectively. Multiple linear regression determined factors (e.g. age, sex, D0 Hb, D0 reticulocyte count, D0 parasitaemia, G6PD status, thalassaemia (grouping homo \& heterozygous $\mathrm{HbE}$ and $\alpha$ and $\beta$ thalassaemia) for the D7-D0 $\mathrm{Hb}$ fall and MAFt (backwardstepwise approach). A linear mixed effects regression model determined factors associated with changes over time in the mean reticulocyte counts, and mean $\mathrm{Hb}$ concentrations; analysis of covariance was adopted to adjust for the $\mathrm{D} 0 \mathrm{Hb}$ concentration by including it as a model covariate. Factors influencing $\mathrm{Hb}$ recovery were determined by logistic regression.

\section{Results}

Patient disposition and baseline characteristics

Of 134 patients volunteering for the trial, 109 were recruited (Fig. 1); the main disqualifying reasons were a negative malaria slide and being G6PDn after that sample size had been reached. Most patients were young male farmers in their 20s (median $23 \mathrm{y}$ ); the other patients were either preschool children or children at school. The youngest patient was 4 years old and pre-adolescent children ( $\leq 12$ y) accounted for $\sim 20 \%$ of the total. Baseline characteristics between the DHAPP and DHAPP+SLDPQ arms were essentially the same (Table 1) aside from significantly different distributions of the platelet counts and LDH concentrations (probable chance findings).

Following treatment, 5 patients were lost to follow up, 2 withdrew early, and 23 had recurrent $P f$ parasitaemias between D12-28, classed as late treatment failures (LTF). LTF rates were not significantly different $(p=0.18)$ between DHAPP+SLDPQ: 9/56 (16.1\%) vs. DHAPP 14/53 (26.4\%) recipients.

Genotyping confirmed 12 G6PD Viangchan patients: 9 hemizygous males and 3 heterozygous females. Eight males and one female were diagnosed correctly by the FST but one male (enzyme activity $3.1 \mathrm{U} / \mathrm{g} \mathrm{Hb}$ ) and two females (7.9 \& 8.4 $\mathrm{U} / \mathrm{g} \mathrm{Hb}$ ) were diagnosed as G6PDn. Three patients ( 1 male, 2 females) who were all FST and RDT G6PDn did not have G6PD activity measurements; the male was classed as G6PDn and the two females were genotyped as G6PD wild type. Additional file 1 lists the G6PD genotype and FST data.

\section{Primary outcome \& D7-D0 changes}

For all patients, the nadir $\mathrm{Hb}$ occurred on $\mathrm{D} 7$ : mean (range) $11.6(6.4-15.6) \mathrm{g} / \mathrm{dL}$. In the DHAPP+SLDPQ treated patients, the mean $\mathrm{Hb}$ was significantly $(p=0.040)$ lower in the G6PDd patients 10.9 (8.5 - 13.2) vs. G6PDn $12.05(8.7-14.5) \mathrm{g} / \mathrm{dL}$. Within the G6PDn group, D7 mean Hbs were significantly lower $(p=0.01)$ in the DHAPP vs. DHAPP+SLDPQ recipients: 11.19 vs. $12.05 \mathrm{~g} /$ $\mathrm{dL}, \Delta=-0.86(-1.56--0.15)$.

The absolute D7-D0 $\mathrm{Hb}$ declines were consistent between the G6PDd and G6PDn patients and inversely related to $\mathrm{D} 0 \mathrm{Hb}$ (Fig. 2): median -1.5 (mean 1.45), interquartile range (IQR) -2.1 - - 0.6 , and range $-4.8-2.4 \mathrm{~g}$ / $\mathrm{dL}$. In the DHAPP+SLDPQ arm, the mean (range) $\mathrm{Hb}$ declines were not significantly $(p=0.63)$ different in the G6PDd vs. G6PDn arms: $-1.46(-3.3-0.4) \mathrm{g} / \mathrm{dL}$ vs. $-1.24(-4.6-2.4) \mathrm{g} / \mathrm{dL}$, for fractional falls of $-11.2(-25.6$ $-3.9) \%$ vs. $-8.6(-25.6-20.7) \%$. 


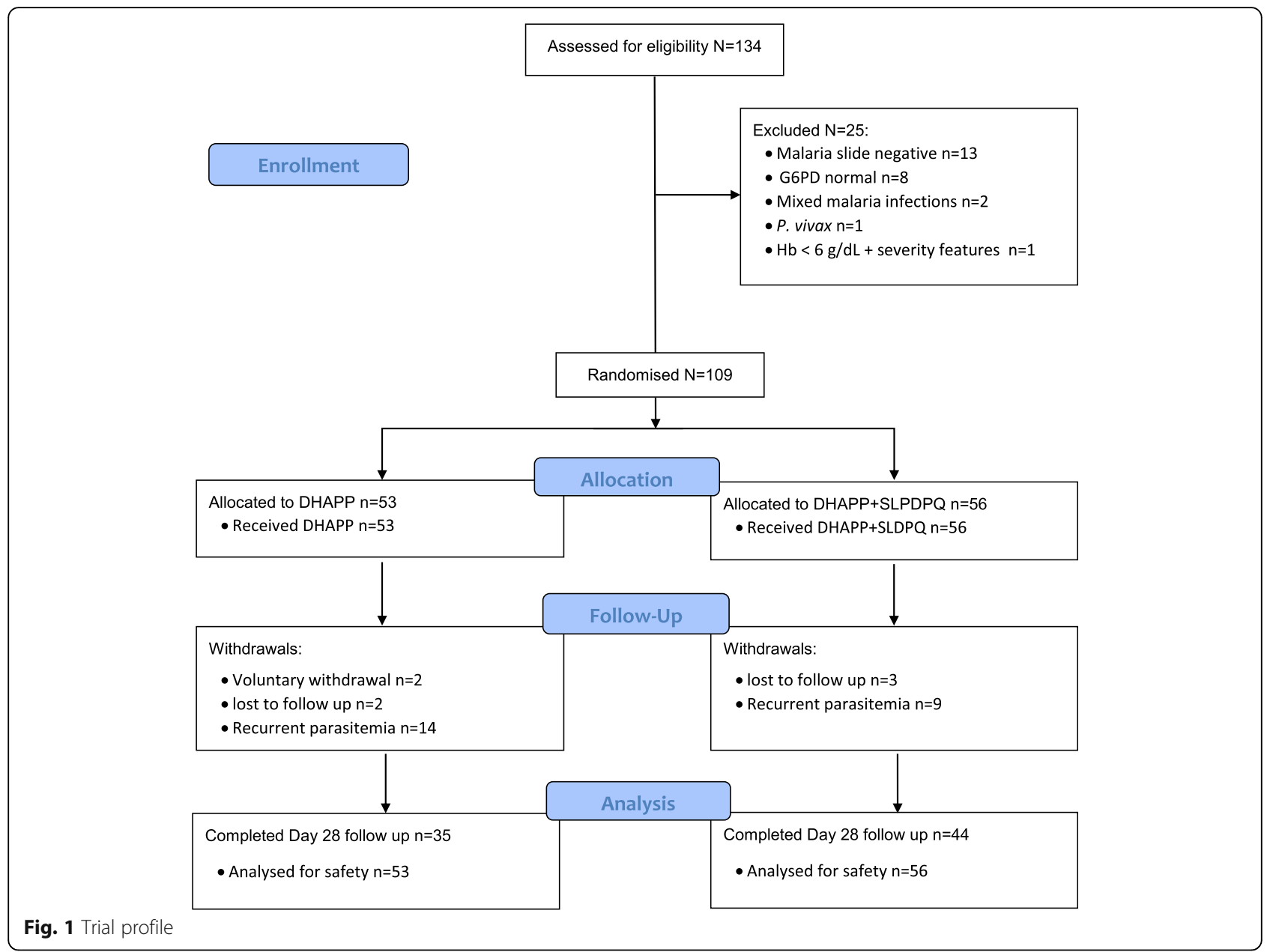

The multiple linear regression model demonstrated an inverse relationship between the D7-D0 decline and (i) $\mathrm{D} 0 \mathrm{Hb}$, and (ii) D0 parasite count (Table 2), but DHAPP + SLDPQ, relative to the DHAPP, was associated a positive change in the D7-D0 $\mathrm{Hb}$, a mean increase of 0.52 (0.14-0.94), $p=0.009$. These three significant factors explain $42.4 \%$ of the variation in the D7-D0 Hb decline (adjusted $\mathrm{R}^{2}=0.4247$ ).

\section{Haemoglobin dynamics}

For all patients, the mean HemoCue measured nadir $\mathrm{Hb}$ was $12.4 \mathrm{~g} / \mathrm{dL}$ (D7) and rose to $13.1 \mathrm{~g} / \mathrm{dL}$ by $\mathrm{D} 28 ; \mathrm{Hb}$ dynamics according to G6PD status are shown in Fig. 3. Being on DHAPP+SLDPQ, male, and of increasing age were associated with higher changes in $\mathrm{Hb}$ whereas reticulocyte dynamics were inversely associated with $\mathrm{Hb}$ dynamics (Table 2).

\section{Malaria attributable fall and $\mathrm{Hb}$ recovery}

Post D7, the MAFt was higher in the G6PDd vs. the G6PDn group but the difference was not significantly different $(p=0.063): 1.81$ vs. $0.57 \mathrm{~g} / \mathrm{dL}, \Delta$
$=-1.25(-0.75-2.56) \mathrm{g} / \mathrm{dL}$. MAFt explanatory fac tors were $\mathrm{D} 7 \mathrm{Hb}$ concentration, treatment failure, both associated with lower a MAFt, and male sex (increased MAFt). The D28 Hb recovery rate was low, 35.7\% (25/98), and more likely in males and older patients but less likely with higher $\mathrm{D} 0 \mathrm{Hb}$ concentrations (Table 2).

\section{Reticulocyte counts}

The mean reticulocyte counts in the G6PDd and normal patients decreased initially on D1-3, increasing thereafter to peak on D7 for the G6PDd group and D14 for the G6PDn group (Fig. 4). The mean baseline reticulocyte count was significantly ( $p=0.01$ ) higher in G6PDd $(2.9 \%)$ vs. G6PDn $(1.8 \%)$ patients. There was an inverse relationship between the $\mathrm{D} 0 \mathrm{Hb}$ and reticulocyte dynamics (Table 2).

\section{Methaemoglobinemia}

There was little change in the median metHb concentrations over time in both treatment arms; the highest value was $3.6 \%$ in a DHAPP-SLDPQ treated G6PDn patient (Additional file 2). Within the G6PDn group, metHb 
Table 1 Baseline characteristics by treatment arm

\begin{tabular}{|c|c|c|c|}
\hline Parameter & DHAPP $\operatorname{arm} N=53$ & DHAPP + SLDPQ arm N = 56 & $P$ value \\
\hline Age in years & $20(14-31)[6-76]$ & $24.5(17.5-32)[4-65]$ & 0.25 \\
\hline Age $\leq 12$ y & $12(22.6)$ & $9(16.1)$ & 0.38 \\
\hline Sex M:F & $44: 9$ & $44: 12$ & 0.56 \\
\hline \multicolumn{4}{|l|}{ Occupation } \\
\hline Farmer & $40(75.5)$ & $46(83.6)$ & 0.29 \\
\hline Symptoms & $n=53$ & $n=55$ & \\
\hline VAS score ${ }^{a}$ & $2(2-2)[2,3]$ & $2(2-2)[1-3]$ & 0.45 \\
\hline Fever & $52(98.1)$ & $53(96.4)$ & 0.58 \\
\hline Chills & $51(96.2)$ & $53(96.4)$ & 0.97 \\
\hline Headache & $49(92.5)$ & $54(98.2)$ & 0.16 \\
\hline Anorexia & $1(1.9)$ & $0(0)$ & 0.31 \\
\hline Nausea & $49(92.5)$ & $41(74.6)$ & 0.01 \\
\hline Vomiting & $51(96.2)$ & $53(96.4)$ & 0.97 \\
\hline Abdominal pain & $4(7.6)$ & $4(7.3)$ & 0.96 \\
\hline Palpitations & $3(5.7)$ & $9(16.4)$ & 0.08 \\
\hline Cough & $3(5.7)$ & $3(5.5)$ & 0.96 \\
\hline Muscle aches & $4(7.6)$ & $7(12.7)$ & 0.37 \\
\hline Passing normal colour urine & $53(100)$ & $55(100)$ & - \\
\hline \multicolumn{4}{|l|}{ Physical signs } \\
\hline Temperature ${ }^{\circ} \mathrm{C}$ & $38(38-38.5)[38-41]$ & $38(38-38.5)$ [38-40] & 0.75 \\
\hline Weight kg & $48(36-55)[17-73]$ & $50.5(40-55.5)[10-74]$ & 0.32 \\
\hline Respiratory rate / minute & $30(26-30)[20-34]$ & $30(28-30)[20-32]$ & 0.58 \\
\hline Normal colour of lips & $53(100)$ & $55(100)$ & - \\
\hline Pale conjunctivae/palms/tongue & $0(0)$ & $0(0)$ & - \\
\hline Palpable liver & $0(0)$ & $0(0)$ & - \\
\hline Palpable spleen & $0(0)$ & $0(0)$ & - \\
\hline \multicolumn{4}{|l|}{ G6PD data } \\
\hline G6PD activity median U/g Hb & 10.45 (7.9-12.9) [0.4-17.9] & $10.15(6.7-11.7)[0.4-25]$ & 0.23 \\
\hline G6PD deficient by FST ${ }^{\mathrm{b}}$ & $3(5.7)$ & $6(10.9)$ & 0.49 \\
\hline G6PD deficient by $\mathrm{RDT}^{\mathrm{c}}$ & $3(5.7)$ & $6(10.9)$ & 0.49 \\
\hline G6PD Viangchan & 3 & $9(42.8)$ & 0.69 \\
\hline Haemoglobin electrophoresis & $n=52$ & $n=54$ & \\
\hline Normal haemoglobin & $23(44.2)$ & $28(51.8)$ & 0.34 \\
\hline Haemoglobin E & $22(42.3)$ & $19(35.2)$ & \\
\hline Mixed $\mathrm{Hb}$ E alpha thalassemia & $3(5.8)$ & $1(1.8)$ & \\
\hline Mixed $\mathrm{Hb} \mathrm{E}$ beta thalassemia & 0 & $3(5.6)$ & \\
\hline Alpha thalassemia & $3(5.8)$ & $3(5.6)$ & \\
\hline Heterozygous foetal haemoglobin & $1(1.9)$ & 0 & \\
\hline \multicolumn{4}{|l|}{ Full blood count } \\
\hline Haemoglobin g/dL & $12.63(2.29)[6.9-18.2]$ & 13.19 (2.1) [8.7-18] & 0.19 \\
\hline Reticulocyte count \% & $1.5(1-2)[0.4-4.2]$ & $1.65(1.05-2.6)[0.3-9.2]$ & 0.13 \\
\hline Methaemoglobin \% & $1.5(1.3-1.8)[0.7-1.9]$ & $1.3(1.2-1.7)[0.6-2]$ & 0.90 \\
\hline Total white cell count $\times 10^{3} / \mu \mathrm{L}$ & $5.6(4.5-7.0)[1.5-13]$ & $5.45(4.45-6.3)[1.6-10.3]$ & 0.50 \\
\hline Platelet count $\times 10^{3} / \mu \mathrm{L}$ & $89(49-122)[12-336)$ & $101.5(76-156.5)[10-328]$ & 0.04 \\
\hline
\end{tabular}


Table 1 Baseline characteristics by treatment arm (Continued)

\begin{tabular}{|c|c|c|c|}
\hline Parameter & DHAPP $\operatorname{arm} N=53$ & DHAPP + SLDPQ arm N = 56 & $P$ value \\
\hline \multicolumn{4}{|l|}{ Biochemical parameters } \\
\hline Total bilirubin $\mu \mathrm{mol} / \mathrm{L}$ & 15.65 (8.6-19.4) [3.7-56.1] & $13.35(10.1-22.1)[3.7-90.5]$ & 0.87 \\
\hline Conjugated bilirubin $\mu \mathrm{mol} / \mathrm{L}$ & $6.35(4.1-9.2)[1.3-16.7]$ & $5.85(4.45-9.25)[2.3-20.4]$ & 0.70 \\
\hline Free bilirubin $\mu \mathrm{mol} / \mathrm{L}$ & $8.2(5.2-10.9)[2.2-49.0)$ & $7.4(5.75-12.5)[1.4-70.1]$ & 0.97 \\
\hline AST IU/L & $26(22-38)[12-133]$ & $28(22-38)[15-84]$ & 0.99 \\
\hline ALT IU/L & $21(16-28)[6-125]$ & $23.5(15.5-35.5)[4-140]$ & 0.42 \\
\hline$L D H I U / L$ & $250(222-347)[137-772]$ & $225.5(200-285)[59-657]$ & 0.04 \\
\hline Haptoglobin g/L & $0.69(0.1-1.43)[0.03-2.24]$ & $0.59(0.1-1.53)[0.01-2.64]$ & 0.60 \\
\hline Creatinine $\mu \mathrm{mol} / \mathrm{L}$ & $63(49-74)[29-112]$ & $63.5(44.5-72)[18-120]$ & 0.53 \\
\hline \multicolumn{4}{|l|}{ Parasite data } \\
\hline Falciparum parasitaemia N/ $\mu \mathrm{L}$ & $19,800(5800-68,119)[1-350,000]$ & $18,600(3923-36,093[40-800,200]$ & 0.40 \\
\hline \multicolumn{4}{|l|}{ Urine } \\
\hline Hillmen colour scale & $3(3-3)[1-4]$ & $3(3-3)[1-4]$ & 0.57 \\
\hline $\begin{array}{l}\text { a VAS - visual analogue scale from } 1 \\
\text { Continuous data are median (interqu } \\
\text { Categorical data are } n(\%) \\
\text { b Fluorescent spot test } \\
\text { c Rapid diagnostic test } \\
\text { Continuous data are median (interqu } \\
\text { Categorical data are } n(\%) \\
\text { Entries in bold are statistically signifi }\end{array}$ & ange], except the haemoglobin con & an (standard deviation) [range] & \\
\hline
\end{tabular}

distributions were significantly different comparing D0 [1.3 (0.6-2)] vs: (i) D3 [1.6 (0.6-3.4)], $p=0.005)$, and (ii) D7 [1.6 (0.9-2.4)], $p=0.007$.

Clinical adverse events and reported symptoms over time All patients tolerated their treatments well. Five patients developed late vomiting on D0, $1.2-2.4 \mathrm{~h}$ post drug administration that was probably drug related: four $($ DHAPP $=1$ ) were aged 7 or 8 with grade 1 (mild) vomiting, and one was a DHAPP+SLDPQ treated, 35 years old male with grade 2 (moderate) vomiting. There were no AEs of clinical concern and no drug-related SAEs. Reported symptoms over time are shown in Additional file 3; no patient complained of itching, developing a rash, or passing dark urine.

Six $(5.9 \%)$ of 102 patients had fractional falls in $\mathrm{Hb} \geq$ $25 \%$ ( $25-33.1 \%$, median $25.5 \%$ ); five were G6PDn (one on DHAPP+SLDPQ) and one was G6PDd on DHAPP + SLDPQ.

\section{Discussion}

We evaluated the WHO recommended dose of SLDPQ in DHAPP-treated Cambodian patients and have shown

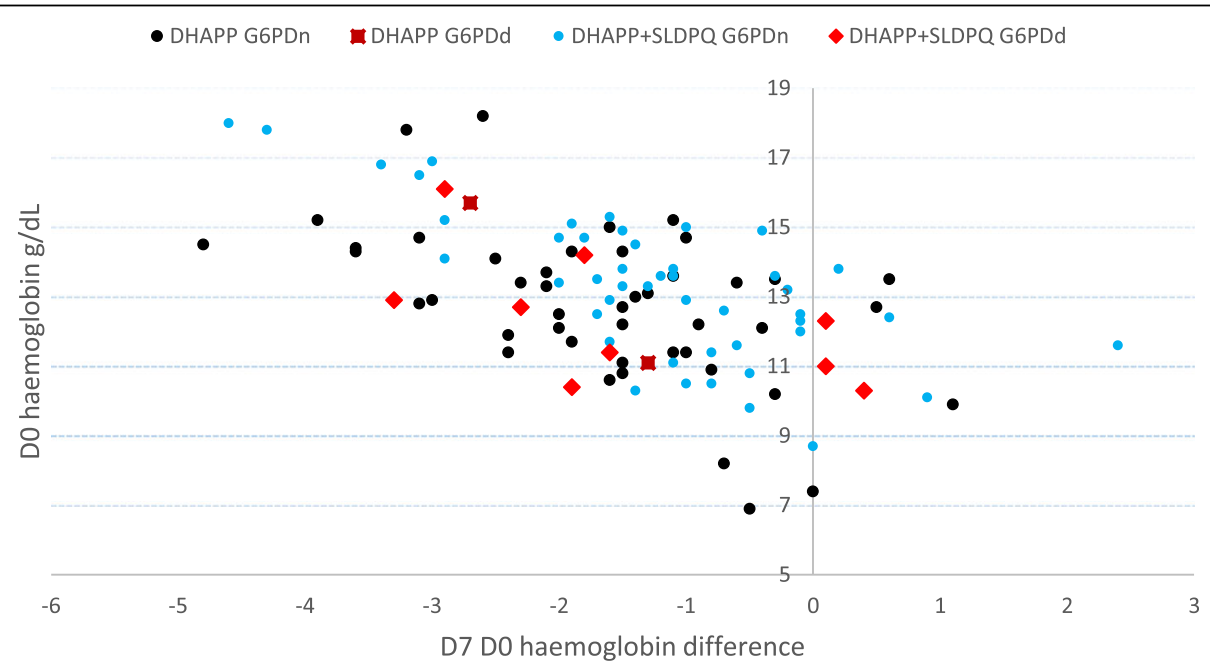

Fig. 2 Scatterplot of baseline hemoglobin concentrations and D7-D0 declines in the patients treated with dihydroartemisinin-piperaquine +/single low dose primaquine, by G6PD status (normal or deficient) 
Table 2 Independent factors associated with several markers of haemoglobin dynamics and recovery in all patients

\begin{tabular}{|c|c|c|c|}
\hline Parameter & Coefficient & 95\% confidence interval & $P$ value \\
\hline \multicolumn{4}{|l|}{$\begin{array}{l}\text { Day } 7 \text { Day } 0 \text { decline in haemoglobin } \\
\text { concentration }\end{array}$} \\
\hline D0 haemoglobin concentration & -0.34 & $-0.45--0.24$ & $<0.001$ \\
\hline D0 parasite count & $-2.69 \times 10^{-7}$ & $-4.81 \times 10^{-6}--5.60 \times 10^{-7}$ & 0.014 \\
\hline On DHAPP+ SLDPQ & 0.48 & $0.08-0.88$ & 0.019 \\
\hline \multicolumn{4}{|l|}{ Haemoglobin dynamics over 28 days } \\
\hline DHAPP+SLDPQ & 0.69 & $0.11-1.27$ & 0.019 \\
\hline Age & 0.034 & $0.014-0.055$ & 0.001 \\
\hline Male & 0.91 & $0.19-1.62$ & 0.013 \\
\hline Reticulocyte dynamics & -0.12 & $-0.17--0.06$ & 0.000 \\
\hline \multicolumn{4}{|c|}{ Total malaria attributable fall in haemoglobin } \\
\hline Treatment failure & -1.28 & $-2.27--0.029$ & 0.012 \\
\hline D7 haemoglobin concentration & -0.53 & $-0.74--0.32$ & $<0.001$ \\
\hline Male & 0.99 & $0.004-1.99$ & 0.049 \\
\hline \multicolumn{4}{|l|}{ Haemoglobin recovery ${ }^{a}$} \\
\hline Male & 7.2 & $1.3-40.0$ & 0.025 \\
\hline Age & 1.04 & $1.001-1.08$ & 0.045 \\
\hline D0 haemoglobin concentration & 0.51 & $0.34-0.76$ & 0.001 \\
\hline \multicolumn{4}{|l|}{ Reticulocyte dynamics } \\
\hline D0 haemoglobin concentration & -0.320 & $-0.420--0.219$ & $<0.001$ \\
\hline
\end{tabular}

it was well tolerated and did not result in clinically significant haemolysis in moderately severe G6PDd Viangchan. Initial declines in $\mathrm{Hb}$ were not significantly different by G6PD status but the DHAPP+SLDPQ treated G6PDd patients had significantly lower mean D7 $\mathrm{Hb}$ by just over $1 \mathrm{~g} / \mathrm{dL}$.
The initial fall in $\mathrm{Hb}$ is the most critical period that determines whether a patient develops clinically severe anaemia to warrant a blood transfusion; in SE Asia, that risk in uncomplicated falciparum malaria is $1 \%$ [11]. The anxiety of malaria control programmes is that this risk would increase if SLDPQ were deployed. There is

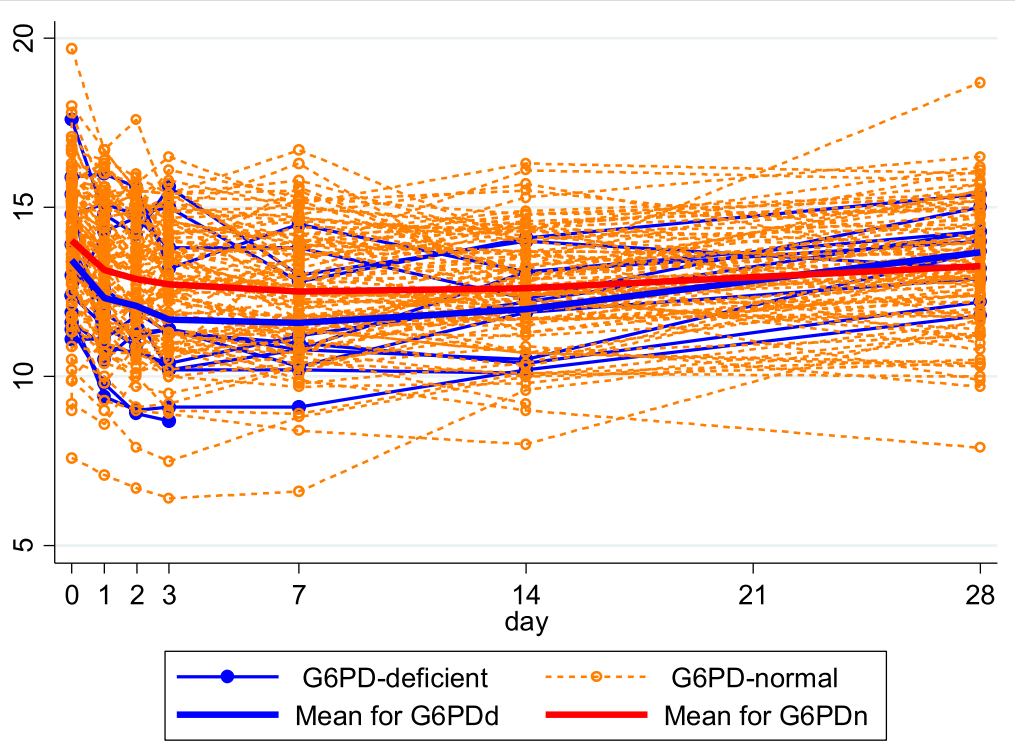

Fig. 3 Haemoglobin dynamics of the HemoCue measured hemoglobin concentrations as a function of glucose-6-phosphate dehydrogenase (G6PD) status 


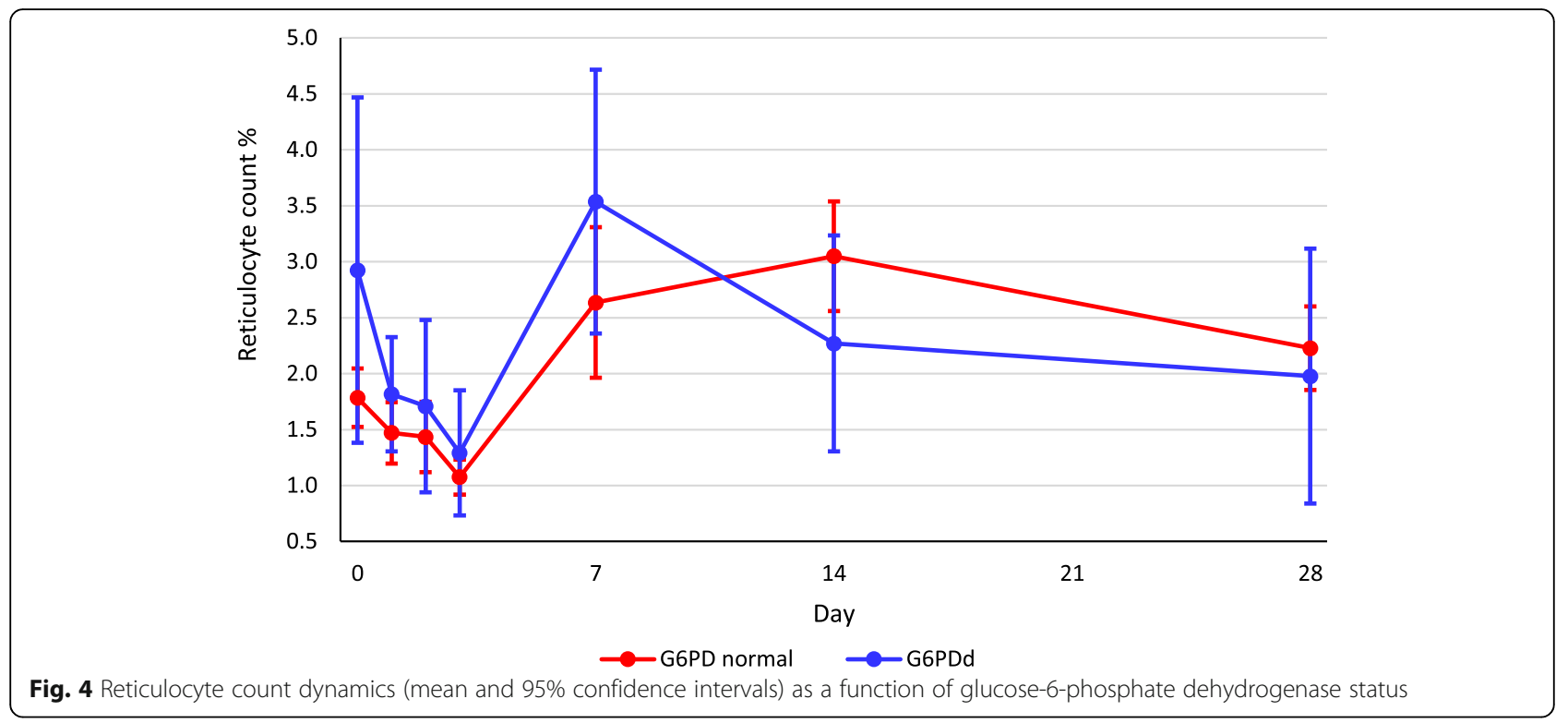

now growing evidence that although G6PDd patients have lower mean nadir $\mathrm{Hb}$ concentrations or greater $\mathrm{Hb}$ declines, these are not clinically threatening $[16,17$, 22, 23].

Factors associated with a greater initial D7-D0 fall in our patients $\mathrm{Hb}$ were higher baseline $\mathrm{Hb}$ concentrations and parasitaemia, consistent with previous studies [10, 11, 30]. A higher parasite biomass results in greater malaria-related intra- and extravascular haemolysis and greater bone marrow suppression [31]. G6PDd was not a factor but this may have been due to small patient numbers. Interestingly, DHAPP+SLDPQ recipients had a lower D7-D0 decline, which is counter intuitive and difficult to explain. Tine et al. also found lower D7-D0 $\mathrm{Hb}$ declines in their adult African patients who received DHAPP+SLDPQ vs. DHAPP alone [18]. The D7 Hb difference $(p=0.01)$ between their G6PDd and G6PDn $\mathrm{ACT}+\mathrm{SLDPQ}$ recipients was $\sim 0.9 \mathrm{~g} / \mathrm{dL}$, a value similar to our study.

After D7, the mean rise in $\mathrm{Hb}$ (measured by the MAFt) was less in patients with LTF and those with a greater initial decline in $\mathrm{Hb}$ but higher in males vs. females. Hb recovery was also more likely in males and patients with higher baseline Hbs. Our high rate of LTF reconfirms the deleterious effect of multidrug resistant $P$. falciparum in the GMS [32-34] and this is probably increasing anaemia in malaria-affected communities. Our LTF rate was unaffected by SLDPQ; indeed, PQ at therapeutic doses does not possess blood stage activity against $P$. falciparum $[35,36]$.

Although G6PDd was not a factor determining MAFt or recovery, the G6PDd patients appear to have had a more rapid rate of $\mathrm{Hb}$ increase and an earlier time to mean peak reticulocytaemia. Bastiens et al detected a trend of a higher D7 reticulocytaemia in their G6PDd vs. G6PDn asymptomatic falciparum carriers treated with 0.25 or $0.4 \mathrm{mg} / \mathrm{kg}$ of $\mathrm{PQ}$ [16]. It is plausible that the G6PDd individuals mount a more robust bone marrow response following PQ but teasing out $\mathrm{PQ}$ vs. malaria related reticulocytosis might be challenging.

Important side effects of PQ are abdominal pain, vomiting and methaemoglobinemia; all are dose dependent and seen more often with doses $\geq 30 \mathrm{mg}$ [37]. Very few patients reported abdominal pain and 5 had late vomiting. The maximum MetHb was $3.6 \%, 3-5$ fold lower than the $\sim 10-20 \%$ level that causes blue lips [38].

The main study limitation was the very small number (12) of G6PDd patients; thus, reducing substantially our statistical power. Nevertheless, we were able to show a significantly lower $\mathrm{D} 7 \mathrm{Hb}$ in DHAPP-SLDPQ treated G6PDd patients. We were only able to recruit three patients with $\mathrm{Hbs} \leq 8 \mathrm{~g} / \mathrm{dL}$ so more data are needed in moderate and severe anaemia because such patients are usually treated in the community. In Cambodia, $\sim 5$ and $\sim 12 \%$ of malaria patients have $\mathrm{Hbs}<5$ and $<7 \mathrm{~g} / \mathrm{dL}$, respectively (analysis from [27]). Our follow up was short - 28 days - because our focus was safety, especially the first week during which the nadir $\mathrm{Hb}$ occurs. This also explains our low rate $(\sim 1 / 3)$ of $\mathrm{Hb}$ recovery, which may have improved with longer follow up $[10,11,30]$. As expected, most recruited patients were young male farmers, an important risk group in the GMS, so our data are applicable to other GMS countries with moderately severe G6PD variants and a similar malaria epidemiological characteristics as Cambodia [5, 39]. More work on SLDPQ is needed in other malaria endemic regions. 


\section{Conclusions}

Our study supports the use of SLDPQ in Cambodia and other GMS countries with similar G6PDd variants. Thanks partly to this study, Cambodia is now deploying SLDPQ and the design of an optimised, age-based SLDPQ regimen should facilitate its deployment down to the village level [40]. SLDPQ deployment should be carried out in conjunction with pharmacovigilance [40].

\section{Additional files}

Additional file 1: List of G6PD status with G6PD enzyme activity (measured in a subset), baseline reticulocyte count and haemoglobin concentration and the result of the fluorescent spot test. (XLSX $13 \mathrm{~kb}$ )

Additional file 2: Methaemoglobin concentration over time, expressed as the \% of total haemoglobin. Within the G6PDn group, metHb distributions were significantly different for: (i) D3 [1.6 (0.6-3.4)] vs. D0 [1.3 (0.6-2)] $p=0.005$ ), and (ii) D7 [1.6 (0.9-2.4)] vs. D0 ( $p=0.007)$. (DOCX $13 \mathrm{~kb})$

Additional file 3: Symptoms reported and selected signs detected during follow up and selected signs suggestive of anaemia. (DOCX $30 \mathrm{~kb}$ )

\section{Abbreviations}

ACTs: artemisinin-based combination therapies; AE: adverse event; AHA: acute haemolytic anaemia; ALT: alanine aminotransferase; AST: aspartate aminotransferase; CRFs: case record forms; D: Day; DHAPP: dihydroartemisinin piperaquine; G6PDd: glucose-6-phosphate dehydrogenase deficiency; GMS: Greater Mekong Sub Region; Hb: haemoglobin; LTF: late treatment failure; MAFt: total malaria attributable fall; metHb: methaemoglobin; NE: northeast; Pf: Plasmodium falciparum; PQ: primaquine; PV: Plasmodium vivax; SAE: serious adverse event; SD: standard deviation; SE: southeast; SLDPQ: single low dose primaquine; WHO: World Health Organisation

\section{Acknowledgements}

We thank the patients for agreeing to participate in this study and the local team in the field who executed the study. We acknowledge Dr. Siddhi Aryal of the Malaria Consortium who liaised with the CDC/PMI. During the early planning phase of this trial, WRJT was supported by the $5 \%$ Initiative (Canal 1) from the French Government.

\section{Funding}

This research was made possible through support provided by the U.S. President's Malaria Initiative via the Office of Health, Infectious Diseases, and Nutrition, Bureau for Global Health, U.S. Agency for International Development, under the terms of an Interagency Agreement with CDC and the Malaria Consortium. The opinions expressed herein are those of the author(s) and do not necessarily reflect the views of the Centers for Disease Control and Prevention or the U.S. Agency for International Development. These organisations played a role in this study, including protocol development, study monitoring, and reviewing this manuscript.

\section{Availability of data and materials}

Selected data generated and analysed during this study are included in this published article and its supplementary information files. Requests for additional data can be made in the first instance to the corresponding author whose institution has a data access committee that considers requests for data.

\section{Authors' contributions}

Core protocol development team - WRJT, DM, LD, SK, SB, ST. Protocol review: LD, MF, JH, CH, ST, AR-F. Study execution \& coordination: LD, SK, WRJT, DM. Laboratory analyses \& coordination: SK, DM, NK. Study monitoring \& oversight - LD, SL, MF, NW, JH, HR. Data analysis \& drafting first manuscript draft -WRJT, MM. Critical review of the manuscript - LD, DM, NW, MF, $J \mathrm{H}$. All authors have read and approved the manuscript, and ensure that this is the case.

\section{Ethics approval and consent to participate}

The Cambodian National Ethics Committee and the U.S. Centers for Disease Control and Prevention and was approved the study, the latter as nonengagement in human subjects research. All subjects or their legal guardians gave written informed consent to participate.

\section{Consent for publication}

Consent to publish was in the patient information sheet / consent from. No addition consent was required.

\section{Competing interests}

The authors declare that they have no competing interests.

\section{Publisher's Note}

Springer Nature remains neutral with regard to jurisdictional claims in published maps and institutional affiliations.

\section{Author details}

${ }^{1}$ National Center for National Centre for Parasitology, Entomology and Malaria Control, Phnom Penh, Cambodia. ${ }^{2}$ School of Public Health, National Institute of Public Health, Phnom Penh, Cambodia. Institut Pasteur du Cambodge, Phnom Penh, Cambodia. ${ }^{4}$ Malaria Consortium, Phnom Penh, Cambodia. ${ }^{5} \mathrm{WHO}$ Cambodia country office, Pasteur Street, Phnom Penh, Cambodia. ${ }^{6}$ Malaria Branch, Centers for Disease Control and Prevention, 1600 Clifton Rd, Atlanta, GA 30333, USA. U.S. President's Malaria Initiative, Malaria Branch, Division Parasitic Diseases and Malaria, Centers for Disease Control and Prevention, Bangkok, Thailand. ${ }^{8}$ U.S. President's Malaria Initiative, Malaria Branch, Division Parasitic Diseases and Malaria, Centers for Disease Control and Prevention, Atlanta, GA, USA. ${ }^{9}$ Mahidol Oxford Tropical Medicine Research unit (MORU), 420/60 Rajvithi Road, Bangkok 10400, Thailand.

${ }^{10}$ Centre for Tropical Medicine, Nuffield Department of Medicine, University of Oxford, Oxford, UK. ${ }^{11}$ Biology of Host-Parasite Interactions Unit, Malaria Genetics and Resistance Group, Institut Pasteur - INSERM U1201 - CNRS ERL9195, Paris, France.

Received: 14 January 2019 Accepted: 1 March 2019

Published online: 12 March 2019

\section{References}

1. Chen I, Poirot E, Newman M, Kandula D, Shah R, Hwang J, Cohen JM, Gosling R, Rooney L. An assessment of the supply, programmatic use, and regulatory issues of single low-dose primaquine as a plasmodium falciparum gametocytocide for sub-Saharan Africa. Malar J. 2015;14:204.

2. Bolchoz L, Budinsky RA, McMillan DC, Jollow DJ. Primaquine-induced hemolytic anemia: formation and hemotoxicity of the arylhydroxylamine metabolite 6-methoxy-8-hydroxylaminoquinoline. J Pharmacol Exp Ther. 2001;297(2):509-15.

3. Bolchoz $\sqcup$, Morrow JD, Jollow DJ, McMillan DC. Primaquine-induced hemolytic anemia: effect of 6-methoxy-8-hydroxylaminoquinoline on rat erythrocyte sulfhydryl status, membrane lipids, cytoskeletal proteins, and morphology. J Pharmacol Exp Ther. 2002;303(1):141-8.

4. Arese P, Gallo V, Pantaleo A, Turrini F. Life and death of Glucose-6phosphate dehydrogenase (G6PD) deficient erythrocytes - role of redox stress and band 3 modifications. Transfus Med Hemother. 2012;39(5):328-34.

5. Matsuoka H, Thuan DT, van Thien H, Kanbe T, Jalloh A, Hirai M, Arai M, Dung NT, Kawamoto F. Seven different glucose-6-phosphate dehydrogenase variants including a new variant distributed in lam Dong Province in southern Vietnam. Acta Med Okayama. 2007;61(4):213-9.

6. Matsuoka H, Wang J, Hirai M, Arai M, Yoshida S, Kobayashi T, Jalloh A, Lin K, Kawamoto F. Glucose-6-phosphate dehydrogenase (G6PD) mutations in Myanmar: G6PD Mahidol (487G>a) is the most common variant in the Myanmar population. J Hum Genet. 2004;49(10):544-7.

7. Kim S, Nguon C, Guillard B, Duong S, Chy S, Sum S, Nhem S, Bouchier C, Tichit M, Christophel E, et al. Performance of the CareStart G6PD deficiency screening test, a point-of-care diagnostic for primaquine therapy screening. PLoS One. 2011;6(12):e28357.

8. Howes RE, Battle KE, Satyagraha AW, Baird JK, Hay SI. G6PD deficiency: global distribution, genetic variants and primaquine therapy. Adv Parasitol. 2013;81:133-201. 
9. Beutler E, Duparc S, Group GPDW. Glucose-6-phosphate dehydrogenase deficiency and antimalarial drug development. Am J Trop Med Hyg. 2007; 77(4):779-89.

10. Taylor WR, Widjaja H, Basri H, Tjitra E, Ohrt C, Taufik T, Baso S, Hoffman SL, Richie TL. Haemoglobin dynamics in Papuan and non-Papuan adults in Northeast Papua, Indonesia, with acute, uncomplicated vivax or falciparum malaria. Malar J. 2013;12:209.

11. Price RN, Simpson JA, Nosten F, Luxemburger C, Hkirjaroen L, ter Kuile F, Chongsuphajaisiddhi T, White NJ. Factors contributing to anemia after uncomplicated falciparum malaria. Am J Trop Med Hyg. 2001;65(5):614-22.

12. Kheng S, Muth S, Taylor WR, Tops N, Kosal K, Sothea K, Souy P, Kim S, Char CM, Vanna C, et al. Tolerability and safety of weekly primaquine against relapse of plasmodium vivax in Cambodians with glucose-6-phosphate dehydrogenase deficiency. BMC Med. 2015;13:203.

13. Charoenlarp P, Areekul S, Harinasuta T, Sirivorasarn P. The haemolytic effect of a single dose of $45 \mathrm{mg}$ of primaquine in G-6-PD deficient Thais. J Med Assoc Thail. 1972:55(11):631-8.

14. Everett WD, Yoshida A, Pearlman E. Hemoglobin E and glucose-6-phosphate deficiency in the Khmer air force (Cambodia). Am J Trop Med Hyg. 1977; 26(4):597-601.

15. Shah SS, Macharia A, Makale J, Uyoga S, Kivinen K, Craik R, Hubbart C, Wellems TE, Rockett KA, Kwiatkowski DP, et al. Genetic determinants of glucose-6phosphate dehydrogenase activity in Kenya. BMC Med Genet. 2014;15:93.

16. Bastiaens GJH, Tiono AB, Okebe J, Pett HE, Coulibaly SA, Goncalves BP, Affara M, Ouedraogo A, Bougouma EC, Sanou GS, et al. Safety of single lowdose primaquine in glucose-6-phosphate dehydrogenase deficient falciparum-infected African males: two open-label, randomized, safety trials. PLoS One. 2018;13(1):e0190272

17. Chen I, Diawara H, Mahamar A, Sanogo K, Keita S, Kone D, Diarra K, Djimde M, Keita M, Brown J, et al. Safety of single-dose Primaquine in G6PDdeficient and G6PD-Normal males in Mali without malaria: an open-label, phase 1, dose-adjustment trial. J Infect Dis. 2018;217(8):1298-308.

18. Tine RC, Sylla K, Faye BT, Poirot E, Fall FB, Sow D, Wang D, Ndiaye M, Ndiaye $J$, Faye $B$, et al. Safety and efficacy of adding a single low dose of Primaquine to the treatment of adult patients with plasmodium falciparum malaria in Senegal, to reduce gametocyte carriage: a randomized controlled trial. Clin Infect Dis. 2017;65(4):535-43

19. Mwaiswelo R, Ngasala BE, Jovel I, Gosling R, Premji Z, Poirot E, Mmbando $B P$, Bjorkman A, Martensson A. Safety of a single low-dose of primaquine in addition to standard artemether-lumefantrine regimen for treatment of acute uncomplicated plasmodium falciparum malaria in Tanzania. Malar J. 2016;15:316.

20. Shekalaghe S, Drakeley C, Gosling R, Ndaro A, van Meegeren M, Enevold A, Alifrangis M, Mosha F, Sauerwein R, Bousema T. Primaquine clears submicroscopic plasmodium falciparum gametocytes that persist after treatment with sulphadoxine-pyrimethamine and artesunate. PLoS One. 2007;2(10):e1023.

21. Shekalaghe $S A$, ter Braak R, Daou M, Kavishe R, van den Bijllaardt W, van den Bosch S, Koenderink JB, Luty AJ, Whitty CJ, Drakeley C, et al. In Tanzania, hemolysis after a single dose of primaquine coadministered with an artemisinin is not restricted to glucose-6-phosphate dehydrogenasedeficient (G6PD A-) individuals. Antimicrob Agents Chemother. 2010;54(5): 1762-8.

22. Goncalves BP, Tiono AB, Ouedraogo A, Guelbeogo WM, Bradley J, Nebie I, Siaka D, Lanke K, Eziefula AC, Diarra A, et al. Single low dose primaquine to reduce gametocyte carriage and plasmodium falciparum transmission after artemether-lumefantrine in children with asymptomatic infection: a randomised, double-blind, placebo-controlled trial. BMC Med. 2016;14:40

23. Bancone G, Chowwiwat N, Somsakchaicharoen R, Poodpanya L, Moo PK, Gornsawun G, Kajeechiwa L, Thwin MM, Rakthinthong S, Nosten S, et al. Single low dose Primaquine $(0.25 \mathrm{mg} / \mathrm{kg})$ does not cause clinically significant Haemolysis in G6PD deficient subjects. PLoS One. 2016;11(3):e0151898.

24. Lin JT, Lon C, Spring MD, Sok S, Chann S, Ittiverakul M, Kuntawunginn W, My M, Thay $K$, Rahman $R$, et al. Single dose primaquine to reduce gametocyte carriage and plasmodium falciparum transmission in Cambodia: an open-label randomized trial. PLoS One. 2017;12(6):e0168702.

25. Smithuis F, Kyaw MK, Phe O, Win T, Aung PP, Oo AP, Naing AL, Nyo MY, Myint NZ, Imwong M, et al. Effectiveness of five artemisinin combination regimens with or without primaquine in uncomplicated falciparum malaria: an open-label randomised trial. Lancet Infect Dis. 2010;10(10):673-81.
26. Heng S, Durnez L, Mao S, Siv S, Tho S, Mean V, Sluydts V, Coosemans M Passive case detection of malaria in Ratanakiri Province (Cambodia) to detect villages at higher risk for malaria. Malar J. 2017;16(1):104.

27. Khim N, Benedet C, Kim S, Kheng S, Siv S, Leang R, Lek S, Muth S, Chea N, Chuor CM, et al. G6PD deficiency in plasmodium falciparum and plasmodium vivax malaria-infected Cambodian patients. Malar J. 2013;12:171.

28. Roca-Feltrer A, Khim N, Kim S, Chy S, Canier L, Kerleguer A, Tor P, Chuor CM, Kheng S, Siv S, et al. Field trial evaluation of the performances of point-of-care tests for screening G6PD deficiency in Cambodia. PLoS One. 2014;9(12):e116143.

29. Hillmen P, Hall C, Marsh JC, Elebute M, Bombara MP, Petro BE, Cullen MJ, Richards SJ, Rollins SA, Mojcik CF, et al. Effect of eculizumab on hemolysis and transfusion requirements in patients with paroxysmal nocturnal hemoglobinuria. N Engl J Med. 2004;350(6):552-9.

30. Sowunmi A, Gbotosho GO, Happi CT, Fateye BA. Factors contributing to anaemia after uncomplicated plasmodium falciparum malaria in children. Acta Trop. 2010;113(2):155-61.

31. Phillips RE, Pasvol G. Anaemia of Plasmodium falciparum malaria. Baillieres Clin Haematol. 1992;5(2):315-30.

32. Imwong M, Hien TT, Thuy-Nhien NT, Dondorp AM, White NJ. Spread of a single multidrug resistant malaria parasite lineage (PfPailin) to Vietnam. Lancet Infect Dis. 2017:17(10):1022-3.

33. Amaratunga C, Lim P, Suon S, Sreng S, Mao S, Sopha C, Sam B, Dek D, Try V, Amato $R$, et al. Dihydroartemisinin-piperaquine resistance in Plasmodium falciparum malaria in Cambodia: a multisite prospective cohort study. Lancet Infect Dis. 2016;16(3):357-65.

34. Witkowski B, Duru V, Khim N, Ross LS, Saintpierre B, Beghain J, Chy S, Kim S, Ke $S$, Kloeung $N$, et al. A surrogate marker of piperaquine-resistant plasmodium falciparum malaria: a phenotype-genotype association study. Lancet Infect Dis. 2017;17(2):174-83.

35. Pukrittayakamee S, Chotivanich K, Chantra A, Clemens R, Looareesuwan S, White NJ. Activities of artesunate and primaquine against asexual- and sexual-stage parasites in falciparum malaria. Antimicrob Agents Chemother. 2004;48(4):1329-34.

36. Arnold J, Alving AS, Hockwald RS, Clayman CB, Dern RJ, Beutler E, Flanagan $\mathrm{CL}$, Jeffery GM. The antimalarial action of primaquine against the blood and tissue stages of falciparum malaria (Panama, P-F-6 strain). J Lab Clin Med. 1955:46(3):391-7.

37. Clayman CB, Arnold J, Hockwald RS, Yount EH Jr, Edgcomb JH, Alving AS. Toxicity of primaquine in Caucasians. J Am Med Assoc. 1952;149(17):1563-8.

38. Wright RO, Lewander WJ, Woolf AD. Methemoglobinemia: etiology, pharmacology, and clinical management. Ann Emerg Med. 1999;34(5):646-56.

39. Bancone G, Chu CS, Somsakchaicharoen R, Chowwiwat N, Parker DM, Charunwatthana P, White NJ, Nosten FH. Characterization of G6PD genotypes and phenotypes on the northwestern Thailand-Myanmar border. PLoS One. 2014;9(12):e116063.

40. Leang R, Khu NH, Mukaka M, Debackere M, Tripura R, Kheang ST, Chy S, Kak $\mathrm{N}$, Buchy $\mathrm{P}$, Tarantola A, et al. An optimised age-based dosing regimen for single low-dose primaquine for blocking malaria transmission in Cambodia. BMC Med. 2016;14(1):171.

Ready to submit your research? Choose BMC and benefit from:

- fast, convenient online submission

- thorough peer review by experienced researchers in your field

- rapid publication on acceptance

- support for research data, including large and complex data types

- gold Open Access which fosters wider collaboration and increased citations

- maximum visibility for your research: over $100 \mathrm{M}$ website views per year

At $\mathrm{BMC}$, research is always in progress.

Learn more biomedcentral.com/submissions 